\title{
Correction
}

Bundesgesundheitsbl 2018 $61: 98$

https://doi.org/10.1007/s00103-017-2679-6

Online publiziert: 7. Dezember 2017

(c) Springer-Verlag GmbH Deutschland, ein Teil von Springer Nature 2017

CrossMark

Maxie Bunz · Hans-Guido Mücke

Abteilung Umwelthygiene, Fachgebiet II, 1.5: Umweltmedizin und gesundheitliche Bewertung,

Umweltbundesamt, Berlin, Deutschland

\section{Correction: Klimawandel - physische und psychische Folgen}

\section{Correction:}

Bundesgesundheitsbl 2017

https://doi.org/10.1007/s00103-017-

2548-3

In der Originalpublikation dieses Beitrags wurde eine Zahl falsch dargestellt. Statt etwa 5000 Menschen, die zusätzlich an den Folgen der Hitzebelastung im Sommer 2003 starben, waren es 7000 Menschen.

Im Folgenden ist der entsprechende Satz im Unterkapitel „Kardiovaskuläre Erkrankungen“, zweiter Absatz, korrekt dargestellt:

Das Hitzeextrem mit stärkstem $\mathrm{Ge}$ sundheitseffekt war der Sommer 2003, in dem europaweit über 50.000 und in Deutschland etwa 7000 Menschen zusätzlich an den Folgen der Hitzebelastung, z. B. an Herzschlag, HerzKreislauf-Erkrankungen und renalen Erkrankungen, starben [1].

Die Korrektur hat keinen Einfluss auf den Text des Unterkapitels resp. das Fazit.

\section{Korrespondenzadresse}

\section{Dr. M. Bunz}

Abteilung Umwelthygiene, Fachgebiet II, 1.5: Umweltmedizin und gesundheitliche Bewertung, Umweltbundesamt Corrensplatz 1, 14195 Berlin, Deutschland maxie.bunz@uba.de

\section{Literatur}

1. Jendritzky G, Koppe C (2008) Die Auswirkungen von thermischen Belastungen auf die Mortalität. In: Lozán JL et al (Hrsg) Warnsignal Klima-Gesundheitsrisiken Gefahren für Pflanzen, Tiere und Menschen. GEO Wissenschaftliche Auswertungen, Hamburg, S149-153 